\title{
Concurrent and Prospective Associations between Metacognition and Depression in Adolescents
}

Jeremy K Russell*1 and John R Seeley ${ }^{2}$

${ }^{1}$ Trillium Behavioral Health, Eugene, USA

${ }^{2}$ Oregon Research Institute, Eugene, USA

\begin{abstract}
Background: Addressing one's metacognitive beliefs is a relatively new, yet promising approach in the treatment of adolescent depression. However, multiple aspects of metacognition can be found in cognitive measures that were created prior to this recent focus.
\end{abstract}

Objectives: The current study leveraged data collected from the Oregon Adolescent Depression Project (OADP) to address a series of questions regarding the associations between metacognitions and adolescent depression.

Methods: Participants included 1,709 community-residing adolescents who were diagnostically assessed on four occasions between the ages of 16 and 30. A construct of metacognition was developed from the battery of assessments in the OADP, following the model established by pioneering metacognitive researcher Adrian Wells. Confirmatory factor analysis demonstrated an acceptable model fit of a four-indicant metacognition construct based on four measures that assessed worry, self-consciousness, self-reinforcement, and mastery.

Results: The metacognition construct was highly correlated with concurrent depression symptoms, with significantly greater associations for female versus male adolescents. Conversely, higher levels of dysfunctional metacognitions in adolescence were predictive of a first incidence of major depressive disorder in emerging adulthood for male but not for female adolescents.

Conclusion: Study findings suggest that therapies that address metacognitive beliefs may be particularly effective in treating depression, especially for female adolescents. In addition, this study indicates metacognition might act as a key marker for identifying youth at risk for the onset of major depressive disorder in emerging adulthood, especially amongst male adolescents.

Keywords: Metacognition; Rumination; Worry; Adolescent; Depression.

\section{Introduction}

Many adolescents struggle with negative cognitions, yet not all struggle with depression. The metacognitive approach to depression, as presented by Wells is built upon the theory that it is the adolescent's metacognitive response to inner experiences that maintains emotional disturbances and gives power to negative cognitions [1]. Likewise, Wells also posits that any internal challenging of negative cognitions is occurring at the metacognitive level. Metacognitions appraise and influence thoughts, emotions, and strategies for coping in both healthy and unhealthy ways. Metacognitions are shown to be unhealthy when operating in a pattern known as the Cognitive Attentional Syndrome (CAS) [1] which consists of worry, rumination, fixed attention, and unhelpful self-regulation strategies or coping behaviors (e.g., "Worrying helps me to avoid a panic attack"). Metacognitions also have the potential to bring awareness, insight, and resilience when recognizing that the content of thoughts do not always correspond to reality and subsequently do not require fixed attention [2], giving the individual the room to explore the value of the thought process itself (e.g., "This line of thinking isn't going to help my situation at all, so I don't need to worry about it right now").

Treatments that focus on metacognitive modes of processing such as Metacognitive Therapy (MCT) show considerable promise for the amelioration of depression [3] as well as cognitive processes that have been shown to exacerbate and maintain depressive symptoms such as worry and rumination [4]. Hoffmann, Sawyer and Morina refer to MCT as a "Third Wave" [5] of Cognitive Behavioral Therapy (CBT) and there are some indications that MCT may be more effective in the treatment of depression and better tolerated than traditional CBT [6]. However, the research regarding the treatment of adolescent depression with MCT is rather limited and traditional CBT remains the most widely used treatment modality for adolescent depression because it has been studied extensively and has been shown to be effective, yet the effects vary widely across studies. According to Spirito et al., there are indications that the effectiveness traditional CBT may have diminished over time [7].

An adolescent boy ruminating on how unlovable he is would likely be asked by his CBT therapist, "What evidence do you have of this?" whereas an MCT therapist would likely ask something like, "Are there advantages to ruminating on this?" CBT therapists' line of questioning, while seeking to assist adolescents by challenging the reality of their perceptions [8], may inadvertently place themselves in the role of adversary to the adolescents' thoughts and feelings. Adolescents, while in the midst of discovering many of the "realities" of childhood are no longer true, and as they are discovering themselves as unique individuals, place greater stock on their thoughts and feelings than on the reality of the situation (e.g., "These thoughts and feelings may be negative and unhelpful, but they are mine, and they are a part of me"). MCT isn't as concerned with the testing of how realistic a thought is or, subsequently, how to deal with such a thought; rather it acknowledges

*Corresponding author: Jeremy Russell, Trillium Behavioral Health 2186 Sally Way, Eugene, USA, Tel: 97401 541-913-3958; Email: jkirkrussell@gmail.com

Received September 24, 2015; Accepted November 11, 2015; Published December 04, 2015

Citation: Russell JK, Seeley JR (2015) Concurrent and Prospective Associations between Metacognition and Depression in Adolescents. J Psychol Abnorm S1: 001. doi:10.4172/jpab.S1-001

Copyright: ( 2015 Russell JK, et al. This is an open-access article distributed under the terms of the Creative Commons Attribution License, which permits unrestricted use, distribution, and reproduction in any medium, provided the original author and source are credited. 
the presence of the thought without judgement, and then proceeds to question the need or the value of engaging with that line of thinking [1]. Subsequently, it stands to reason that MCT has significant potential to be an effective treatment with adolescents, and is in need of further investigation.

A primary focus of MCT is the CAS and the metacognitive beliefs that continue to reactivate the syndrome. The CAS is predominantly exhibited by excessive and sustained worrying and rumination. Such lines of thinking and dwelling often have an attentional bias which lock on to perceived potential threats [1]. The repeated practice of worry, for example, can potentially lead to a sense of uncontrollability and inevitability of such lines of thinking, which, when accompanied with greater accessibility of threat concepts, can lead to superstitious thinking and/or feelings of punishment [9]. This meta-worry, along with other metacognitive beliefs can persist even after the adolescent has learned ways to manage or ameliorate worrying, which makes them strong candidates as potential contributors to the relapse or recurrence of depressive episodes.

With 50-70\% of adolescents with an episode of depression remitting within 5 years [10] and with 30\% remitting following CBT [11], any treatment for adolescent depression should include considerable attention towards the prevention of recurrence, yet there is still a considerable knowledge gap in this area [12]. However, Singer and Dobson [13] found that individuals at risk for depression relapse who were trained in the metacognitive skill of acceptance had considerably less negative attitudes towards negative experiences than those who ruminated or those who used distraction coping methods. MCT also shows promise in the amelioration of cognitive processes that are considered to be maintaining or causal factors in depression such as rumination and metacognitive beliefs $[3,14]$.

Metacognitions assessment such as the Meta-Cognitions Questionnaire-Adolescent Version (MCQ-A) [15] also has considerable potential of detecting when an adolescent is more likely to relapse as it is structured to detect the CAS in adolescents, assessing such precursor variables as rumination, the uncontrollability of thoughts, a lack of cognitive confidence, and worry about a perceived inevitable situation. The MCQ-A is a 30 -item measure that was developed to assess metacognitive beliefs in adolescents. The MCQ-A measures thought processes and beliefs about thinking in relation to worry and intrusive thoughts and includes five factors: positive beliefs; uncontrollability and danger; cognitive confidence; superstition, punishment, and responsibility; and cognitive self-consciousness.

One important aspect of adolescent depression, which is also a major focus of MCT is rumination. Rumination has been defined by Nolen-Hoeksema as "repetitive and passive thinking about one's symptoms of depression and the possible causes and consequences of these symptoms." [16] Papageorgiou and Wells also developed a scale to assess positive beliefs about rumination [17] and showed relationships between positive and negative metacognitive beliefs, rumination, and depression [18]. In their meta-analysis of self-focused attention, Mor and Winquist concluded that of all the forms found in the literature, rumination had the strongest and most consistent relationship with depressive symptoms [19]. Nolen-Hoeksema explains that ruminators focus much of their metacognitive thoughts on the problems in their lives, subsequently making it difficult for them to find good solutions to those problems, keeping them passive and hopeless [20].

The distinctive gender differences in the etiology of adolescent depression is shown in many studies [21,13], however there is still little known about the determinants of these differences, even though such determinants would be of great clinical significance. One of the strongest candidates for such a determinate is rumination as it has been shown in multiple studies that female adolescents tend to ruminate more than males [22,20] However, the explanations for these differences and why they occur in certain situations but not in others remains largely speculative. When this speculation does occur, it is predominantly focused on female rumination; attention on the reduced levels of rumination in boys is minimal and the focus on when rumination does occur in boys appears almost non-existent.

These differences in rumination are speculated [23] to be a result of greater pressure on girls in early childhood to yield and take the other's perspective in times of conflict and to be more other-directed. Mor and Winquist posit that this pressure to put others before the self can potentially lead to a hyper-focus on the lives and problems of others which can translate at a metacognitive level to a hyper-focus on her own problems [20]. This increased attention on girls is also, in large part, due to the fact that girls after puberty are twice as likely to experience depression, a fact which has left depression and it's determinants in adolescent boys under-studied and under-served [13,23].

With metacognition at the core of self-consciousness, and as an integral part of the development and the refining of the perception of the self, it stands to reason that some of the effects of changes in metacognitive processes may take years to unfold. In fact, a number of articles studying metacognitive theory suggest replicating such studies with a much larger population [6] and over a longer time period [24,23]. The current study leverages data collected from the Oregon Adolescent Depression Project (OADP) [25] to create a metacognition construct in order to address the following questions:

1. Is the metacognition construct stable over time?

2. What is the magnitude of association between metacognition and symptoms of depression, both concurrently and prospectively?

3. Are there differences in metacognition between current cases of major depressive disorder (MDD), remitted cases of MDD, and adolescents with no history of MDD?

4. Is metacognition assessed during adolescence predictive of the first onset and recurrence of major depressive disorder in adulthood?

5. Are the associations moderated by sex?

\section{Methods}

\section{Participants}

We used data from the Oregon Adolescent Depression Project (OADP) [17], a longitudinal study of high school students who were assessed twice during adolescence, a third time at approximately age 24, and a fourth time at approximately age 30 . Participants were randomly selected for the initial assessment from nine senior high schools representative of urban and rural districts in western Oregon. A total of 1,709 adolescents (mean age $16.6, S D=1.2$ ) completed the initial $\left(\mathrm{T}_{1}\right)$ assessment between 1987 and 1989. The participation rate at $\mathrm{T}_{1}$ was $61 \%$.

Approximately one year later, 1,507 of the adolescents (88\%) returned for a second evaluation $\left(\mathrm{T}_{2}\right)$. Differences between the sample and the larger population from which it was selected, and between participants and those who declined to participate at $\mathrm{T}_{2}$, were small.

All adolescents with a history of psychopathology by $\mathrm{T}_{2}(n=644)$ 
Citation: Russell JK, Seeley JR (2015) Concurrent and Prospective Associations between Metacognition and Depression in Adolescents. J Psychol Abnorm S1: 001. doi:10.4172/jpab.S1-001

Page 3 of 7

and a random sample of adolescents with no history of psychopathology by $\mathrm{T}_{2}(n=457)$ were invited to participate in a third $\left(\mathrm{T}_{3}\right)$ evaluation. All non-white $\mathrm{T}_{2}$ participants were retained to maximize ethnic diversity. Of the $1,101 \mathrm{~T}_{2}$ participants selected for a $\mathrm{T}_{3}$ interview, $941(85 \%)$ completed the age 24 evaluation. At age 30 , all $\mathrm{T}_{3}$ participants were asked to complete the $\mathrm{T}_{4}$ assessment. Of the 941 who participated in the $\mathrm{T}_{3}$ evaluation, 816 (87\%) completed the $\mathrm{T}_{4}$ assessment.

Diagnostic measures: At $\mathrm{T}_{1} \mathrm{~T}_{2}$ and $\mathrm{T}_{3}$, participants were and Schizophrenia for School-Age Children (K-SADS) [26], which combined features of the Epidemiologic and Present Episode versions, and included additional items to derive Diagnostic and Statistical Manual of Mental Disorders, $3^{\text {rd }}$ edition revised [27] diagnoses. Follow-up assessments at $\mathrm{T}_{2}$ and $\mathrm{T}_{3}$ were jointly administered with the Longitudinal Interval Follow-Up Evaluation (LIFE) [28]. The K-SADS/ LIFE procedure provided information regarding the onset and course of disorders since the previous interview. The $\mathrm{T}_{4}$ interview consisted of a joint administration of the LIFE and the Structured Clinical Interview for DSM-IV (SCID) [29] to probe for new or continuing episodes since $T_{3}$. Diagnoses were based on DSM-III-R criteria for $T_{1}$ and $T_{2}$ and DSM-IV criteria for $\mathrm{T}_{3}$ and $\mathrm{T}_{4}$. Interviews at $\mathrm{T}_{3}$ and $\mathrm{T}_{4}$ were conducted by telephone, which generally yields comparable results to face-to-face interviews [17] Diagnostic interviewers had advanced degrees in a mental health field and had completed a 70-hour course in diagnostic interviewing. At each of the four evaluations, audiotapes of a randomly selected sample of interviews were rated by another diagnostician. Interrater reliabilities for diagnoses were good to excellent $[17,30]$.

Three diagnostic groups were created based on current and lifetime histories of MDD at T1: no history of $\operatorname{MDD}(n=1,394)$, a past diagnosis of MDD (remitted cases; $n=273$ ), and a current diagnosis of MDD (current cases; $n=42$ ). To examine the first onset of MDD in adulthood, the reference sample include participants who were followed through T4 (age 30), but had not history of MDD before 19 years of age $(n=532)$. To examine the recurrence of MDD in adulthood, the reference sample included participants who were followed through age 30 , had a history of MDD before 19 years of age and recovered by age $19(n=266)$. interviewed with a version of the Schedule for Affective Disorders

\section{Cognitive measures}

The following measures are presented in Table 1:

The self-consciousness: 23 -item scale assesses three aspects of selfconsciousness: public, private, and social anxiety. The OADP assessed 9 of the 23 items across all 3 subscales. The Self-Consciousness scale is reliable with a test-retest correlation of 0.80 . The scale was also shown [31] to be correlated to the Metacognitions Questionnaire - 30, the adult version of the MCQ-A. This scale is consistent with Factor 1 and 5 of the MCQ-A, positive thoughts about worry and cognitive selfconsciousness, as many of these items are related to worry or reflections upon the self with the potential of a positive outcome as a result.

The self-reinforcement questionnaire [32]: This is a 30-item scale designed to measure self-reinforcement in meaningful settings. This scale assesses the attitudes towards the self in relation to achievements and failures. Internal consistency of this measure was good (alpha $=$ .77) The OADP assessed 10 of the 30 items. It has been shown by Heiby that individuals with low frequency of self-reinforcement are more likely to become depressed than individuals in other groups [33]. Selfreinforcement has also been shown to have a mediated relationship with social anxiety as assessed by the Self-Consciousness Scale [34]. The Self-reinforcement scale overlaps well with Factor 4 of the MCQ-A; superstition, punishment, and responsibility; as both deal with the "I should..." statements surrounding one's thoughts and worries and the perceived consequences of failure.

The worry assessment (5 items) used in the OADP was adapted from the Maudsley Obsessional-Compulsive (MOC) Inventory [35] in order to be more suitable for adolescents. Internal consistency for the MOC subscales were acceptable (alpha $=.70$ to .80 ). The Metacognitions Questionnaire was developed in relation to Generalized Anxiety Disorder and higher scores have been been shown in multiple studies $[36,37]$ to be associated with higher levels of OCD symptoms. The Worry assessment is closely related to Factor 2 of the MCQ-A as both assess the uncontrollability and the danger of worry and worried thinking.

The personal mastery assessment included 3 items selected from The Meta-cognitions Questionnaire (MCQ-A)

"I usually worry about making a good impression."

"I'm concerned about what other people think of me."

"I'm always trying to figure myself out."

"I reflect about myself a lot."

"I'm constantly examining my motives."

The Self-reinforcement Questionnaire

"If I didn't criticize myself frequently, I would continue to do things poorly forever."

"I don't often think positive thoughts about myself."

"I have such high standards for what I demand of myself that I rarely meet those standards."

The Worry assessment (e.g. MOC Inventory)

"I frequently find myself worrying about something."

"I find that almost every day I am upset by unpleasant thoughts that come to mind against my will."

"Sometimes some unimportant thought will run through my mind and bother me for days."

I am presently worrying over possible misfortune.

The Mastery Scale

"I have little control over the things that happen to me."

"Sometimes I feel that I'm being pushed around in life."

"My efforts will determine what my life will be like 5 years from now." me to solve problems."

"Worrying helps me to solve

"I pay close attention to the way my mind works.

"I think a lot about my thoughts."

"I constantly study my thoughts."

The Meta-cognitions Questionnaire (MCQ-A)

"If I could not control my thoughts, I would not be able to function."

"I am constantly aware of my thinking."

"I should be in control of my thoughts all of the time."

The Meta-cognitions Questionnaire (MCQ-A)

"My worrying thoughts persist, no matter how I try to stop them."

"I cannot ignore my worrying thoughts."

"When I start worrying, I cannot stop."

"If I did not control a worrying thought, and then it happened, it would be my fault."

The Meta-cognitions Questionnaire (MCQ-A)

"Not being able to control my thoughts is a sign of weakness."

"I will be punished for not controlling certain thoughts."

"Worrying helps me to avoid problems in the future."

Table 1: Measures Compared with the MCQ-A 
the 7-item Personal Mastery scale [38]. These 3 items were selected to act as a brief assessment of generalized expectations about the degree to which one can direct the events in one's life. Internal consistency for the Personal Mastery scale was acceptable (alpha= 0.79). The 3 items selected overlap with the superstition, punishment, and responsibility factor of the MCQ-A as they both delve into the sense of uncontrollability and inevitability of negative events occurring and a sense that there is an external, invisible hand or force directing these events. Such increased accessibility of threat concepts arise when worry progresses towards more problematic meta-worry [32].

To determine whether the four measures could be combined into a single metacognition construct, a confirmatory factor analysis (CFA) was performed using maximum-likelihood estimation based on the T1 scale scores. Several indicators were used in the evaluation of model fit and interpreted in conjunction with critical cut points recommended by $\mathrm{Yu}[39]$ and $\mathrm{Hu}$ and Bentler [40]. Fit indicators (with associated cutoff values for denoting good fit) were: chi-square test of model fit $(p>$ .05 ), comparative fit index (CFI; $\geq .96$ ), Tucker-Lewis fit index (TLI; $\geq .95$ ), root mean square error of approximation (RMSEA; $\leq .05$ ), and standardized root mean square residual (SRMR; <.08). All four of the measures had significant standardized factor loadings on the single metacognition factor $(\mathrm{p}<.001$, standardized estimates $>.35)$. Four of the five model fit statistics indicated a good fit for the single-factor metacognition construct: chi-square $(2, \mathrm{~N}=1,709)=6.06, \mathrm{p}=.048$; $\mathrm{CFI}=.995 ; \mathrm{TLI}=.984 ; \mathrm{RMSEA}=.034 ;$ and SRMR $=.012$. Given the acceptable model fit, the four metacognition measures were combined by standardizing each of the measures and computing the mean score at both $\mathrm{T} 1$ and $\mathrm{T} 2$ such that higher values indicated greater cognitive dysfunction at each time point.

Depressive symptoms: The 21-item Beck Depression Inventory (BDI) [41] was used to assess current depressive symptomatology. The coefficient alpha of this scale was .88 .

\section{Data analyses}

Pearson correlations were used to evaluate the association between the metacognition construct and depressive symptoms as well as

\begin{tabular}{|c|c|c|c|c|}
\hline & \multicolumn{2}{|c|}{ Time1 } & \multicolumn{2}{c|}{ Time 2 } \\
\hline & Metacognition & BDI & Metacognition & BDI \\
\hline \multicolumn{5}{|c|}{ Time 1 } \\
\hline Metacognition & 1.000 & .658 & .580 & .416 \\
\hline BDI & .493 & 1.000 & .385 & .464 \\
\hline \multicolumn{5}{|c|}{ Time 2 } \\
\hline Metacognition & .637 & .367 & 1.000 & .619 \\
\hline BDI & .311 & .441 & .485 & 1.000 \\
\hline
\end{tabular}

Note: $\mathrm{BDI}=$ Beck Depression Inventory. Correlation coefficients for the female sample are reported above diagonal; correlation coefficients for the male sample are reported below the diagonal. All p-values $<.001$.

Table 2: Concurrent and Prospective Correlations between Metacognitions and Depressive Symptoms

Assessed at Time 1 and Time 2 by Sex. one-year stability of the metacognition measure; the Fisher's $r$ to $\mathrm{z}$ transformation was used to ascertain whether or not the strength of the correlations differed for female and male adolescents. Analysis of variance (ANOVA) was used to compare the T1 diagnostic groups on the metacognition construct measures; pairwise group comparisons were conducted using the Scheffé post hoc test. Logistic regression (LR) models were used to predict the first onset and recurrence of MDD in adulthood. Potential moderating effects of sex were also examined by including interaction terms in the ANOVA and LR models as well as testing the models separately for female and male participants.

\section{Results}

\section{Concurrent and prospective associations between metacognitions and depressive symptoms}

The current and prospect associations between the metacognition construct and BDI assessed at $\mathrm{T} 1$ and $\mathrm{T} 2$ are presented in Table 2; correlation coefficients reported above the diagonal are for the female participants and those reported below the diagonal are for male participants. As can been seen, the metacognitions construct was strongly correlated with the BDI scores at T1 and T2 for female and male participants. However, a stronger association between the metacognition was found for the female sample compared to the male sample at both $\mathrm{T} 1$ (female $\mathrm{r}=0.658$, male $\mathrm{r}=.493 ; \mathrm{r}$ to $\mathrm{z}=5.14, \mathrm{p}<$ 0.001 ) and $\mathrm{T} 2$ (female $\mathrm{r}=0.619$, male $\mathrm{r}=0.485 ; \mathrm{r}$ to $\mathrm{z}=2.37, \mathrm{p}=0.018$ ). Metacognitions at $\mathrm{T} 1$ was moderately predictive of BDI scores at T2 (female $\mathrm{r}=0.416$, male $\mathrm{r}=.311 ; \mathrm{r}$ to $\mathrm{z}=2.31, \mathrm{p}=0.021$ ). The T1-T2 one-year stability of the metacognition construct was strong for both female and male participants $(\mathrm{r}=.636$ and .580 , respectively) and was significantly higher than the stability of the BDI for both sexes (female $\mathrm{r}=.441, \mathrm{r}$ to $\mathrm{z}=7.83, \mathrm{p}<.001$; male $\mathrm{r}=.464, \mathrm{r}$ to $\mathrm{z}=4.18, \mathrm{p}<.001$ ).

\section{MDD diagnostic group differences in metacognitions during adolescence}

The three diagnostic groups created based on current and lifetime histories of MDD at T1 (no MDD history, $\mathrm{n}=1,394$; remitted MDD cases, $n=273$; and current MDD cases, $n=42$ ) were compared on the metacognition construct at T1. A 3 (diagnostic group) X 2 (sex) ANOVA model yielded a significant main effect for diagnostic group $(\mathrm{F}[2,1703]=66.95, \mathrm{p}<.001)$ and $\operatorname{sex}(\mathrm{F}[1,1703]=17.18, \mathrm{p}<.001)$; the two-way interaction between diagnostic group and sex was not significant $(\mathrm{F}[2,1703]=1.41, \mathrm{p}=.245)$. Because the interaction with sex was not significant, the ANOVA model was re-specified without including sex as a factor. The results of the one-way ANOVA along with the descriptive statistics for the metacognition construct by diagnostic group are reported in Table 3. To further examine gender differences, one-way ANOVA results are also reported for male and female samples. For the total sample, each of the three groups differed significantly from each other based on the Scheffe post hoc comparisons in which the no MDD history group had the lowest mean score (-0.09), the remitted MDD cases had an intermediate mean score (0.34), and the current MDD cases had the most elevated mean score (0.75). A similar pattern

\begin{tabular}{|c|c|c|c|c|c|c|c|c|}
\hline \multirow{3}{*}{ Sample } & \multicolumn{6}{|c|}{ Diagnostic Group } & \multirow{3}{*}{ F-ratio } & \multirow{3}{*}{ df } \\
\hline & \multicolumn{2}{|c|}{ No MDD } & \multicolumn{2}{|c|}{ Past MDD } & \multicolumn{2}{|c|}{ Current MDD } & & \\
\hline & M & SD & M & SD & M & SD & & \\
\hline Total & $-0.09_{a}$ & 0.65 & $0.34_{b}$ & 0.71 & $0.75_{c}$ & 0.60 & 76.19 & 2,1706 \\
\hline Female & $-0.02 a$ & 0.67 & $0.40_{b}$ & 0.73 & $0.69_{b}$ & 0.57 & 40.42 & 2,888 \\
\hline Male & $-0.15_{a}$ & 0.63 & $0.20_{b}$ & 0.65 & $0.88_{c}$ & 0.67 & 27.16 & 2,815 \\
\hline
\end{tabular}

Note. $\mathrm{M}=$ mean; $\mathrm{SD}=$ standard deviation; $\mathrm{df}=$ degrees of freedom. Means with different subscripts within the same row differ significantly at $\mathrm{p}<.05$ based on the Scheffe post hoc test. All $\mathrm{F}$ ratios significant at $\mathrm{p}<.001$.

Table 3: Descriptive Statistics and ANOVA Results for Diagnostic Group Comparisons on Metacognitions at Time 1 for the Total, Female, and Male Samples. 
emerged for the female and male samples, however, the remitted MDD cases did not differ significantly from the current MDD cases for the female sample.

\section{Prospective associations between adolescent metacognitions and the first incidence and recurrence of MDD and in early adulthood}

To examine whether metacognitions assessed at T1 predicted the first onset of MDD in adulthood (age 19 to 30), a sequential LR model was tested in which the sex was entered into model as the first step, metacognition construct was entered in the second step, and the interaction between metacognitions was entered in the final step. Of the 532 participants without a history of MDD by age 19 years, $193(36 \%)$ experienced their first MDD episode during the 19-30 age period. In the initial step of the LR model, sex significantly predicted the first onset of MDD in early adulthood, Improvement chi-square $(1, \mathrm{~N}=$ $532)=26.58, \mathrm{p}<.001$, Generalized $\mathrm{R}=.184$, odd ratio $(\mathrm{OR})=2.58$ (95\% confidence interval $[\mathrm{CI}]=1.78-3.72)$. In step 2 , metacognitions significantly predicted MDD first incidence, Improvement chi-square $(1, \mathrm{~N}=532)=9.46, \mathrm{p}=.002$, Generalized $\mathrm{R}=.104, \mathrm{OR}=1.63(95 \% \mathrm{CI}$ $=1.19-2.24)$. In the final step, the interaction between metacognition and sex did not significantly predict MDD first onset, Improvement chisquare $(1, \mathrm{~N}=532)=2.71, \mathrm{p}=.100$. However, sex-specific LR analyses yielded a significant metacognition effect in predicting first incidence for male participants (chi-square $[1, \mathrm{~N}=336]=10.53, \mathrm{p}=.001$; $\mathrm{OR}=$ $2.24,95 \% \mathrm{CI}=1.36-3.68$; Generalized $\mathrm{R}=.165)$ but not for female participants (chi-square $[1, \mathrm{~N}=480]=1.63, \mathrm{p}=.202$; $\mathrm{OR}=1.31,95 \%$ $\mathrm{CI}=0.87-1.97$; Generalized $\mathrm{R}=.000)$

With respect to predicting MDD recurrence in early adulthood, of the 266 participants who experienced and recovered from an episode of MDD by age 19 years, 163 (61\%) experienced MDD recurrence during the 19-30 age period. Following the same sequential LR approach above, neither the main effects for metacognitions and sex nor the interaction term significantly predicted MDD recurrence (all p's > .05). Sex-specific LR analyses yielded non-significant metacognition effects in predicting MDD recurrence for female and male samples (p's > .05).

\section{Discussion}

The four scales selected to measure the metacognition construct were based on indicators that align with the literature on metacognition, as well as similarities between individual items, sub-scales, and the assessments as a whole with the MCQ-A. Given the acceptable model fit for the four cognitive scales as well as the strong correlations the composite measure showed over time for both males and females, the construct created for the purposes of the current study appears to be a valid measure of metacognitive beliefs and the CAS in adolescents.

The finding of the high one-year stability of the metacognition construct, which was considerably more stable than the BDI scores, suggests that metacognition may be more of a trait-like risk factor as opposed to state specific. This finding supports the notion of certain aspects of metacognition such as rumination which act as a coping strategy, albeit an ineffective one, and may be repeatedly activated by other metacognitive factors such as low confidence in problem solving [17] or positive beliefs about rumination[18]. Yet rumination is also predictive of impaired problem solving, thinking, and ultimately conditions such as depression, anxiety, and substance abuse [42]. As a result, ruminators can remain in a cycle of passivity and despair [20] even when depressive symptoms are at low levels, yet this cycle maintains a level of general psychological distress [6] which can then set the stage for a depressive episode. In response, MCT targets this cycle, in particular, for the prevention of depression relapse [6] and shows particular potential as a transdiagnostic treatment to prevent the recurrence of multiple conditions [6].

The metacognition construct was strongly correlated with concurrent BDI scores at both $\mathrm{T} 1$ and $\mathrm{T} 2$ for male adolescents; the correlations were stronger for female adolescents at both times. In addition, metacognition assessed at $\mathrm{T} 1$ was moderately correlated with BDI assessed at T2 for both sexes. However, metacognition significantly predicted the first incidence of MDD in emerging adulthood only for male participants.

The finding of the strong, concurrent association between metacognition and depression is consistent with the current literature but is stronger than the association between the Children's Depression Inventory and the MCQ-A reported by Cartwright-Hatton et al. (2004) [43]. However, metacognition in adolescence did not predict the recurrence of depression in emerging adulthood.

The findings in this study related to the gender differences in the association between metacognition and depression are of particular interest. The association between concurrent metacognition and depression was significantly stronger for females than males. This is contrary to most studies on metacognition, which frequently find little or no significant difference between the sexes. However, this finding is in keeping with literature on rumination, a corollary of metacognition, which shows that adolescent girls are much more likely to ruminate than adolescent boys $[44,45]$ which has been attributed to the female preponderance of depression. Such differences can be attributed to differing social pressures in childhood and adolescence. If there is greater pressure on girls in early childhood to yield and take the other's perspective in times of conflict, then this can lead to a hyper-focus on the lives and problems of others which can translate at a metacognitive level to a hyper-focus on her own problems [46]. These social pressures may translate a certain degree of social acceptance of the female's rumination, whereas these same social pressures respond to such open expression of emotional thinking amongst boys as a sign of weakness. Subsequently, an adolescent boy may need to find for coping with psychological distress more socially acceptable strategies boys, such as distraction. This, in turn, may end up working out better for the boy as this strategy is more effective in coping with depressive symptoms.

Differences in coping strategies may also result in differences in the types of metacognitive factors used to manage perceived stressors. Typically, amongst the metacognitive studies, which have small populations, there is little to no gender difference detected in the subject's metacognitive response. This lack of gender differences detected may be a result of a lack of attempts to split the population as it is already small, or as a result of such gender differences being more difficult to detect as males often are a small minority in such studies. However, one study [32] found that males displayed significantly higher scores in the two metacognitive factors: negative beliefs about thoughts, including themes of superstition, punishment, and responsibility; and cognitive self-consciousness. Differences in metacognitive responses have been shown to relate to symptoms of depression differently, yet there is still considerable need for further research in this area.

Another noteworthy finding is the stronger concurrent associations between metacognition and depression symptoms for females compared to the significant predictive association between metacognition and the onset of MDD in early adulthood for only the male participants. The non-significant predictive association for females runs counter to what would be expected from the previous research and the stronger concurrent association found amongst females. If, as discussed, 
males are more likely to display the metacognitive factors of negative beliefs about thoughts and cognitive self-consciousness, then this may act to mediate the relationship between rumination and depressive symptoms [4]. Also, these metacognitive factors may lead to attempts to control or suppress unwanted thoughts [32]. This suppression of unwanted thoughts may defer psychological stressors for a time, yet paradoxically, it is this suppression that may even act to produce the very preoccupation that was being avoided [47]. Therefore, it is plausible that for some adolescent boys with high levels of metacognition, yet who find such metacognitive processes to be a sign of weakness may find ways to distract themselves and subsequently avoid a depressive episode. However, this deferred maintenance can only last so long and, as the boy enters adulthood with the social pressures less pronounced, and as he spends more time alone within his own thoughts, these metacognitive processes may return in full force setting the stage for a major depressive disorder. This hypothesis is also supported by the differences in attributional styles between males and females. Attributional styles is a concept that has considerable crossover with metacognition as it is apparent that there are metacognitive beliefs intertwined with how one chooses to make attributions. It has been shown that males are more likely than females to take a pessimistic attributional style towards the stability of an event. In other words, males may be more likely to see the cause of a negative event as being consistent over time [46]. Subsequently, if an adolescent boy accepts the negative cognition, "I just can't get ahead in life" he may be better able than his female counterparts to set aside and distract himself from this thought, yet he is more likely to hold on to this belief throughout adolescence and into adulthood. Then, when he finds himself in a place where his life has not gone as planned, he already has a long list of events at his disposal, which can be used to confirm the negative cognition from his childhood, greatly heightening the psychological stress of the situation. Accordingly, MCT shows considerable therapeutic potential as it doesn't seek to challenge this lengthy list of apparent confirmations of the negative cognition, rather it questions the value and the purpose of entertaining such a negative cognition.

For adolescents struggling with depression, females showed more maladaptive attributional patterns which may, in turn, prolong depression symptoms and episodes. This may partially account for the considerably higher relationship between metacognition and concurrent depression levels for females. These maladaptive attributional styles and increased levels of rumination $[47,46]$ amongst females both have the potential to prolong depression symptoms which may partially account for the depression rates for females to be twice that of males. This considerably larger prevalence of depression amongst adolescent girls has led to the increased attention of depression research on adolescent girls, yet this has left depression and it's determinants in adolescent boys under-studied and under-served [47,27]. Clearly, more research is needed in this regard.

\section{References}

1. Wells A (2011) Metacognitive therapy for anxiety and depression. Guilford press.

2. Teasdale JD, Moore RG, Hayhurst H, Pope M, Williams S, et al. (2002) Metacognitive awareness and prevention of relapse in depression: empirical evidence. Journal of consulting and clinical psychology 70: 275

3. Wells A, Fisher P, Myers S, Wheatley J, Patel T, et al. (2009) Metacognitive therapy in recurrent and persistent depression: A multiple-baseline study of a new treatment. Cognitive Therapy and Research 33, 291-300.

4. Papageorgiou C, Wells A (2003) An empirical test of a clinical metacognitive model of rumination and depression. Cognitive therapy and research, 27: 261273.

5. Hofmann SG, Sawyer AT, Fang A (2010) The empirical status of the "new wave" of cognitive behavioral therapy. Psychiatr Clin North Am 33: 701-710.

6. Normann N, van Emmerik AA, Morina N (2014) The efficacy of metacognitive therapy for anxiety and depression: a meta-analytic review. Depress Anxiety 31: 402-411

7. Spirito A, Esposito-Smythers C, Wolff J, Uhl K (2011) Cognitive-behavioral therapy for adolescent depression and suicidality. Child and adolescent psychiatric clinics of North America 20: 191-204.

8. Beck AT, Rush AJ, Shaw BF, Emery G (1979) Cognitive therapy of depression, Guilford New York 152.

9. Fenigstein A, Scheier MF, Buss AH (1975) Public and private self-consciousness: Assessment and theory. Journal of consulting and clinical psychology 43: 522.

10. Dunn V, Goodyer IM (2006) Longitudinal investigation into childhood- and adolescence-onset depression: psychiatric outcome in early adulthood. $\mathrm{Br} J$ Psychiatry 188: 216-222.

11. Dimidjian S, Hollon S D, Dobson K S, Schmaling K B, Kohlenberg R J et al. (2006) Randomized trial of behavioral activation, cognitive therapy, and antidepressant medication in the acute treatment of adults with major depression. Journal of consulting and clinical psychology 74: 658 .

12. Thapar A, Collishaw S, Pine DS, Thapar AK (2012) Depression in adolescence. Lancet 379: 1056-1067.

13. Singer AR, Dobson KS (2007) An experimental investigation of the cognitive vulnerability to depression. Behav Res Ther 45: 563-575

14. Wells A, Fisher P, Myers S, Wheatley J, Patel T, et al. (2012) Metacognitive therapy in treatment-resistant depression: a platform trial. Behav Res Ther 50: 367-373.

15. Cartwright-Hatton S, Mather A, Illingworth V, Brocki J, Harrington R, et al.(2004) Development and preliminary validation of the Meta-cognitions QuestionnaireAdolescent Version. Journal of Anxiety Disorders 18: 411-422.

16. Nolen-Hoeksema S (1991) Responses to depression and their effects on the duration of depressive episodes. Journal of abnormal psychology 100: 569.

17. Papageorgiou C, Wells A (2002) Positive beliefs about depressive rumination: Development and preliminary validation of a self-report scale. Behavior Therapy 32: 13-26.

18. Papageorgiou C, Wells A (2003) An empirical test of a clinical metacognitive mode of rumination and depression. Cognitive therapy and research 27: 261-273.

19. Mor N, Winquist J (2002) Self-focused attention and negative affect: a metaanalysis. Psychological bulletin 128: 638 .

20. Nolen-Hoeksema S (2004) The Response Styles Theory. Depressive rumination 107.

21. Piccinelli M, Wilkinson G (2000) Gender differences in depression. Critical review. Br J Psychiatry 177: 486-492.

22. Morrow J, Nolen-Hoeksema S (1990) Effects of responses to depression on the remediation of depressive affect. J Pers Soc Psychol 58: 519-527.

23. Broderick PC, Korteland C (2004) A prospective study of rumination and depression in early adolescence. Clinical Child Psychology and Psychiatry 9 : 383-394.

24. Spada MM, Caselli G, Manfredi C, Rebecchi D, Rovetto F, et al. (2012) Parenta overprotection and metacognitions as predictors of worry and anxiety. Behav Cogn Psychother 40: 287-296.

25. Lewinsohn PM, Roberts RE, Seeley JR, Rohde P, Gotlib IH, et al. (1994) Adolescent psychopathology: II. Psychosocial risk factors for depression. J Abnorm Psychol 103: 302-315.

26. Orvaschel H, Puig-Antich J, Chambers W, Tabrizi MA, Johnson R (1982) Retrospective assessment of prepubertal major depression with the KiddieSADS-e. J Am Acad Child Psychiatry 21: 392-397.

27. Spitzer RL (2001) Values and assumptions in the development of DSM-III and DSM-III-R: an insider's perspective and a belated response to Sadler, Hulgus, and Agich's "On values in recent American psychiatric classification". J Nerv Ment Dis 189: 351-359.

28. Keller MB, Lavori PW, Friedman B, Nielsen E, Endicott J et al. (1987) The Longitudinal Interval Follow-up Evaluation: A Comprehensive Method for Assessing Outcome in Prospective Longitudinal Studies. Arch Gen Psychiatry 44: $540-548$ 
Citation: Russell JK, Seeley JR (2015) Concurrent and Prospective Associations between Metacognition and Depression in Adolescents. J Psychol Abnorm S1: 001. doi:10.4172/jpab.S1-001

29. Spitzer, R, Williams, J, Gibbon M, First M (1990) Structured Clinical Interview for DSM-III-R (SCID-P/SCIP-NP), (Patient Edition/Non-patient Edition), American Psychiatric Press, Inc, Washington, DC.

30. Rohde P, Lewinsohn PM, Seeley JR, Klein DN, Andrews JA, et al. (2007) Psychosocial functioning of adults who experienced substance use disorders as adolescents. Psychol Addict Behav 21: 155-164

31. Cartwright-Hatton S, Wells A (1997) Beliefs about worry and intrusions: The Meta-Cognitions Questionnaire and its correlates. Journal of anxiety disorders 11: $279-296$

32. Heiby EM (1982) A self-reinforcement questionnaire. Behav Res Ther 20: 397 401.

33. Heiby EM (1983). Depression as a function of the interaction of self-and environmentally controlled reinforcement. Behavior Therapy 14: 430-433.

34. Kocovski NL, Endler NS (2000) Social anxiety, self-regulation, and fear of negative evaluation. European Journal of Personality 14: 347-358.

35. Hodgson RJ, Rachman S (1977) Obsessional-compulsive complaints. Behav Res Ther 15: 389-395.

36. Solem S, Myers SG, Fisher PL, Vogel PA, Wells A (2010) An empirical test of the metacognitive model of obsessive-compulsive symptoms: replication and extension. Journal of anxiety disorders 24: 79-86.

37. Matthews L, Reynolds S, Derisley J (2007) Examining cognitive models of obsessive compulsive disorder in adolescents. Behavioural and Cognitive Psychotherapy 3S1: 002-163.

38. Spencer SM, Patrick JH (2009) Social support and personal mastery as protective resources during emerging adulthood. Journal of Adult Development 16: 191-198.

39. Yu C-Y (2002) Evaluating cutoff criteria of model fit indices for latent variable models with binary and continuous outcomes: Unpublished doctoral dissertation, University of California, Los Angeles.

40. Hu L, Bentler PM (1999) Cutoff criteria for fit indexes in covariance structure analysis: Conventional criteria versus new alternatives. Structural Equation Modeling 6: 1-55.

41. Beck AT, Ward CH, Mendelson M, Mock J, Erbaugh J (1961) An inventory for measuring depression. Archives of General Psychiatry 4: 561-571.

42. Nolen-Hoeksema S, Wisco BE, Lyubomirsky S (2008) Rethinking Rumination. Perspect Psychol Sci 3: 400-424.

43. Cartwright-Hatton S, Mather A, Illingworth V, Brocki J, Harrington R, Wells A (2004) Development and preliminary validation of the Meta-cognitions Questionnaire-Adolescent Version. Journal of Anxiety Disorders 18: 411-422.

44. Morrow J, Nolen-Hoeksema S (1990) Effects of responses to depression on the remediation of depressive affect. J Pers Soc Psychol 58: 519-527.

45. Mor N, Winquist J (2002) Self-focused attention and negative affect: a metaanalysis. Psychological bulletin 128: 638-662.

46. Gladstone TR, Kaslow NJ, Seeley JR, Lewinsohn PM (1997) Sex differences, attributional style, and depressive symptoms among adolescents. J Abnorm Child Psychol 25: 297-306.

47. Wegner DM, Schneider DJ, Carter SR, White TL (1987) Paradoxical effects of thought suppression. Journal of personality and social psychology 53: 5 .
This article was originally published in a special issue, Children Psychology handled by Editor(s). Dr. Gérard Mercier, University of Besancon, France 